\title{
A Methodology and Associated CAD Tools for Support of Concurrent Design of MEMS
}

\author{
B. F. Romanowicz', M. H. Zaman, S. F. Bart, V. L. Rabinovich, I. Tchertkov, \\ C. Hsu, and J. R. Gilbert \\ Microcosm Technologies, Inc., 215 First Street, Suite 219, Cambridge, MA 02142 U.S.A.
}

Key words: MEMS, microsystem, CAD, macro-model, simulation

\begin{abstract}
Development of micro-electro-mechanical systems (MEMS) products is currently hampered by the need for design aids, which can assist in integration of all domains of the design. The cross-disciplinary character of microsystems requires a top-down approach to system design which, in turn, requires designers from many areas to work together in order to understand the effects of one sub-system on another. This paper describes current research on a methodology and tool-set which directly support such an integrated design process.
\end{abstract}

\section{INTRODUCTION}

Considerable progress in technologies for microsystems fabrication has made over the past two decades, resulting in a large variety of commercially successful devices. These products have benefited from the high performance and low manufacturing costs characteristic of MEMS batch fabrication technologies. Though the manufacturing technologies are derived from microelectronic fabrication techniques, most devices require application specific fabrication steps, which must be developed, and characterized. This often results in costly and time consuming prototyping. It

\footnotetext{
'bfr@memcad.com
}

The original version of this chapter was revised: The copyright line was incorrect. This has been corrected. The Erratum to this chapter is available at DOI: 10.1007/978-0-387-35498-9_57 
is recognized that standardization of processes will help reduce the entry cost of microsystems.

MEMS sensors and actuators can be viewed as the electronic interface to the physical world. Some physical quantity of interest is transformed into an electrical quantity that can be measured, or some electrical signal is converted into some action on the environment. As a result of the small dimensions of these transducers, and the semiconductor materials often used, strong field coupling is present, and needs to be taken into account in the modeling of these devices, to accurately capture their behavior. Historically, most of the modeling and simulation effort has gone into developing tools that capture the intricate physics present in MEMS devices.

All MEMS systems have some common layers to their design. These include device design (design a manufacturable component), package design (design a practical package), and system design (design and improve the system the device fits into). The requirement for design aids is best illustrated by considering the MEMS products that have been commercially successful to date. Examples include inkjet printer nozzles, pressure sensors, and a variety of inertial sensors used primarily in the automotive field. In all of these products, the design criteria for each of the individual domains were met successfully in an economic and manufacturable manner.

Development of MEMS products is currently hampered by the need for design aids, which can assist in integration of all domains of the design [1,2]. The cross-disciplinary character requires a top-down approach to system design which, in turn, requires designers from many areas to work together in order to understand the effects of one sub-system on another. What is required is a design methodology based on concurrent design in all required domains. These design domains include the MEMS/MOEMS device, the analog sensing circuitry, the high-level system electronics, the application specific package, and manufacturing sensitivity analysis.

The ability of MEMS devices to be integrated with signal conditioning circuitry and batch fabrication offers an important advantage over their macroscopic counterparts. To ensure proper functioning of such an integrated system, one must perform system-level simulation. Such systemlevel modeling is extremely useful in determining operation characteristics and verifying performance before the device is actually manufactured. This can reduce the need for prototype fabrication and test iterations and significantly reduce cost and time-to-market.

Performing full 3-D physical simulation within each time step of a typical system simulator (such as $\mathrm{SABER}{ }^{\mathrm{TM}}, \mathrm{MATLAB}^{\mathrm{TM}}$, or SPICE) is prohibitively time-consuming and numerically impractical. Hence, in order to simulate the appropriate system level dynamic behavior efficiently, a reduced-ordered model or "macro-model" of the MEMS subsystem must be 
obtained and employed in the system-level simulator. Thus, macro-model construction is a key part of a design methodology [3].

As micro-systems become more complex and the need for models with large numbers of coupled degrees-of-freedom (DOFs) increases, the use of automated tools for generating macro-models becomes increasingly important. Although macro-modeling techniques have been reported by some researchers ([4], [5], [6], [7], [8], [9]), currently there is no systematic method for generating macro-models for MEMS devices in an automatic way.

Design tools to support this methodology must allow passing of models from one group to another to foster communication between them. These tools must also be usable by engineers who are not high level scientists with years of MEMS simulation experience.

In this paper we summarize some of the past three years work on developing methods and tools to enable the concurrent design of MEMS and MST systems. First we describe the concurrent design methodology itself, with particular attention to who does the designing and how different designers interact. We follow that with discussions of a research projects which now provide tools to enable the key (thick) arrow of Figure 1. This is a tool for automatic extraction of dynamic macro-models of MEMS devices.

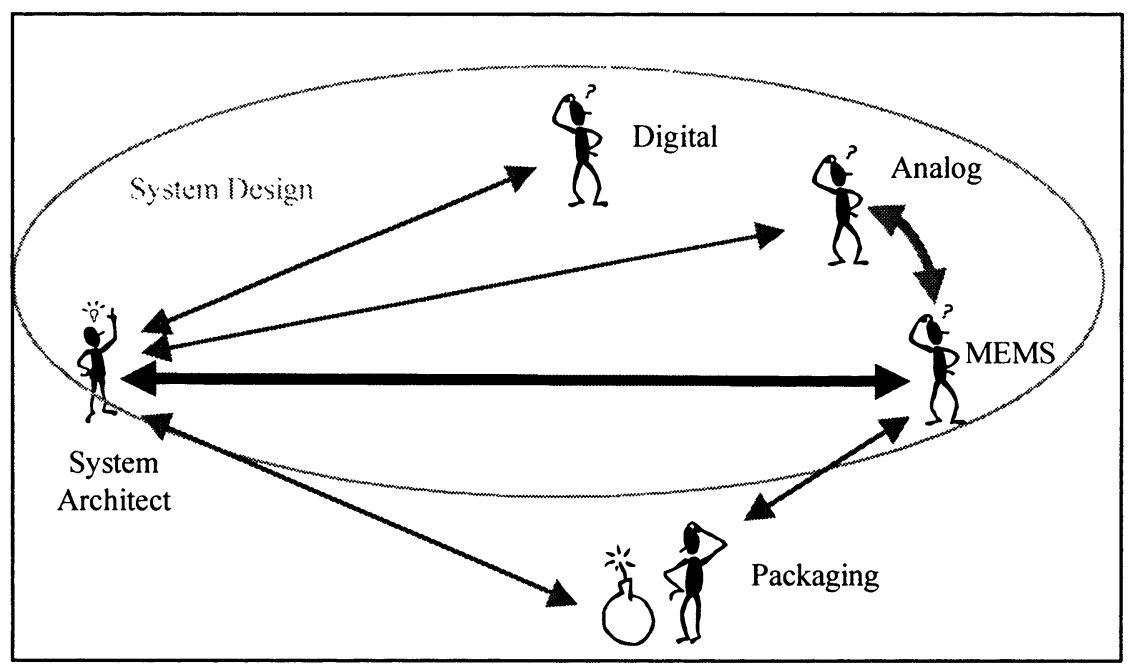

Figure 1. Design groups involved in MEMS design 


\section{CONCURRENT DESIGN OF MEMS}

MEMS design involves several layers of design work, and potentially concurrent engineering among several groups. An "Actor" based view of such concurrent engineering is sketched in Figure 1. A System Architect coordinates the design of a product, drawing on the knowledge and experience of design specialists in the digital and analog circuit, MEMS device, and packaging fields. Successful design of systems containing MEMS components requires a top-down approach to system design. This involves supporting the actors (design groups) in Figure 1 with behavioral modeling and simulation at the level of the System Architect, and enabling the System Architect to specify subsystem functions in each area, by specifying behavioral models.

The use of electronic design automation (EDA) tools for the system level simulation of MEMS is attractive, as complete systems may be simulated together with the physical transducers, analog and digital signal processing, compensation and control modules, and external environmental influences. The System Architect can investigate the effects of various design partitions, and trade off the complexity of the various subsystems, and verify if these options are compatible with the required system performance and functions.

\subsection{Use of HDLs for Design Intent Communication}

Modern analog and mixed signal hardware description languages (HDLs) can advantageously be used to facilitate this communication between the design specialists. In our case, this corresponds to enabling the thick arrows in Figure 1. The use of HDLs, as opposed to SPICE models, for system level simulation supports the use of energy-based physical models. This prevents the introduction of spurious energy sources, which can occur,for example, by using SPICE polynomial sources to model nonlinear elements. HDLs also provide a rich set of syntax, constructs, and modeling methods [10]. These are needed to describe the complex coupled physics present in transducers in an accurate and efficient manner. Signal flow modeling, a technique is often used for the analysis of systems containing feedback loops, is supported by HDLs. This is the approach used by tools such as the SimulinkTM (in MatlabTM). The advantage of using a circuit simulator is the ability to seamlessly introduce models of electronics in the feedback loop. 


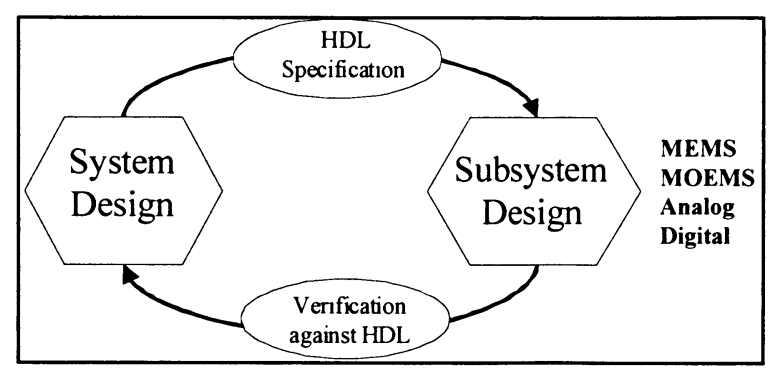

Figure 2. Use of HDLs for specification and validation by system architect

HDLs also support various levels of abstraction, which may be introduced by the System architect as he refines the system from an initial set of components with simplified behavior, to more sophisticated models which contain detailed information about the performance of subsystems, as provided by the various actors.

The System Architect specifies the targeted functionality of each subsystem, as needed to fulfill the required system performance, using VHDL-AMS or a proprietary HDL such as MastTM, VerilogATM, SpectreHDLTM, HDL-ATM, etc. He can perform a system level simulation with these behavioral models to validate the system performance, and validate design partitioning that he has made. For example, it is possible to trade off complexity in the design of a physical transducer against the complexity in the signal processing subsystem. The System Architect then passes each of these behavioral models to the design specialists in each field. These behavioral models are in effect design specifications, which the subsystem designer must now meet. This design process is illustrated in Figure 2.

Each of the design specialists may now work independently on his subsystem to insure that it meets the targeted functionality that is required of it. An example of such a design process, and the CAD tools that support it, is presented in Figure 3. A MEMS inertial sensor subsystem designer receives a specification from the System Architect. After drafting a set of layouts corresponding to the process he wishes to use in manufacturing the device, he then performs an accurate 3D simulation to capture the physics, which are of importance in his design. He then automatically extracts parameters of importance in this design, to create a reduced order model in a HDL of his choice. This behavioral macro-model can now be simulated and compared to the abstract specification provided by the system architect, to verify if the device design provides the required functionality. 


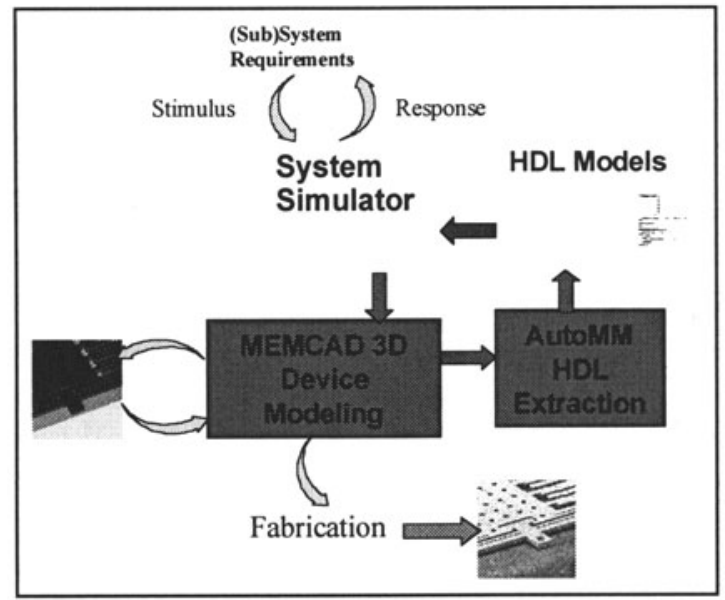

Figure 3. MEMS device design inside the system design loop

The above process is interactive. If any of the actors are unable to meet a targeted specification, they provide the System Architect with a behavioral model of their best design. The System Architect may then change the specification of another subsystem, in order to compensate for the actual performance of a subsystem. This design process is iterated upon until an optimal design is obtained. These decision criteria may include technical specifications such as performance, accuracy, speed, size, power consumption, functionality, but can also include economic and marketing considerations such as cost, reliability, time to market, dimensions. Costly and time consuming prototyping steps are thus avoided.

This top-down approach to MEMS design does place considerable constraints on the CAD tools, as well as on the foundries and plants where the devices are to be manufactured. Without accurate knowledge of parameters such as material properties, residual stresses, manufacturing repeatability, operating conditions, packaging stress, etc. a CAD system can not operate in a predictable manner. In an analogous manner to electronic design automation (EDA), MEMS CAD requires the existence of foundry, process, and even run specific data to predict product performance with an acceptable accuracy. This implies the existence of systematic process characterization through test structures, and the calibration of the CAD tools using constitutive properties extracted from these test structures.

\subsection{System Design Partitioning Issues}

As indicated in Figure 1, MEMS devices are composed of multiple subsystems, which are designed separately and must be integrated. This requires 
not only communication between the sub-system designers as previously discussed, but also an integrated view of how to partition the sub-systems for optimal cost and performance. Integrated or single chip MEMS are usually manufactured using a set of pre and post CMOS micromachining steps using fab compatible materials. An example of an integrated system is the Analog Devices automobile airbag accelerometer [11]. Integrated devices are attractive because of their generally low unit cost in mass production. The integration of electronics close to sensors also helps noise performance.

Hybrid MST systems offer a greater choice of technologies and materials for the physical transducer, thus offering possible advantages in performance. They are however potentially more costly to package and assemble, and signal conditioning may be problematic as parasitic impedance's are introduced between the sensor and front-end electronics. Ford Microelectronics uses a two-chip hybrid system for their airbag accelerometer [12].

Efficient communication between the actors in Figure 1 is required for both a single chip approach, and for a hybrid multi-chip system solution. The interaction between the designers will be simpler in the second case, as they will not (necessarily) be sharing the same silicon die. The exchange of energy and information between subsystems on different chips will take place through the various interconnects (electrical, fluidic, optical, etc.) connecting the separate substrates. Here, the specifications provided by the System Architect will reflect the structural partitioning provided by the multi-chip approach. In both cases however, the CAD support tools supporting the design flow will be similar.

In both integrated or hybrid MEMS, electronics are used to improve the sensor performance by linearization schemes, active feedback, thermal compensation, chopping to reduce $1 / \mathrm{f}$ noise etc. Other system level electronics are often required such as sensor calibration, self-test, programmability and other application specific functions. Additionally design trade-offs are required such as the choice between integrating an onchip pre-amp versus a complete A/D converter. The partitioning of these sub-systems can have a significant effect on the system performance. System level modeling is critical to optimizing this partitioning.

In inertial measurement systems it is possible to trade complexity between the mechanical and electronic subsystems. A cheap and nonlinear sensing element may be improved through linearization and feedback, but this implies additional IC real estate. For example, a closed-loop system may be used to remove all mechanical and geometric non-linearity's in an accelerometer by sensing the position of the seismic mass and applying a correction force equivalent to the acceleration, thereby immobilizing the mass at it's point of rest. Care must be taken here to ensure that mechanical 
resonance frequencies are not excited by capacitive sensing means, which requires a mechanical-electronic co-design. This type of force-feedback design was used in initial accelerometer designs [12]. When improved system modeling demonstrated the linearity of the mechanical part, the feedback was discarded in favor of a smaller, cheaper circuit [13].

Another example occurs in resonant sensors such as MEMS gyroscopes. Here it is possible to trade off mechanical complexity in the form of the resonator $\mathrm{Q}$ versus the phase accuracy of the sensing electronics. The tradeoff point can be very dependent on the specifics of the design (including the fabrication process constraints) and requires modeling to optimize.

All of these design tradeoffs can be taken into account through efficient top-down behavioral model generation and bottom-up validation procedures. Examples of products whose design is supported in MEMCAD today include:

- Inertial Sensors

- Pressure Sensors

- Mirrors, Gratings, Optical Switches

- Electrical/RF Switches

- Thermal Actuators/Sensors
- Packaging Analysis

- Ink Jets

- uTAS and Lab-On-Chip Applications

- Flow Sensors

- Data Storage

\section{EXTRACTION OF MACRO-MODELS}

In this section, we describe a systematic method for modeling the class of electro-mechanical micro-systems that can be represented as multicomponent, lumped, mass-spring-dashpot structures. Examples include accelerometers, gyros, and other structures that have rigid masses and compliant springs. In this lumped modeling assumption, the lumped spring effect originates from mechanical reaction forces and moments of the suspensions (or tethers) holding the proof-mass. Damping forces result from multiple energy loss mechanisms, but are dominated by gas viscosity. In addition, there are electrostatic forces and torques exerted on the dielectrically separated conductors in the system when voltages are applied. The accuracy of the developed method is verified by comparison of two plate-tether MEMS structures to results obtained from the developed models with those from full 3-D physics simulations. Good accuracy is demonstrated in both spatial-domain and frequency-domain dynamic behavior of the models. 


\subsection{Overview}

A semi-automatic and complete modeling procedure that automates the generation of component-level macro-models of MEMS devices has been developed [14]. The user assembles the system-level model by connecting individual component-level macro-models together. For simplicity, the developed method assumes that while the tethers provide mechanical compliance, they are electrostatically inert and massless. It also assumed that the proof mass is electrostatically driven and moves as a rigid body. Devices that do not move as a rigid body, such as membrane devices cannot be accurately modeled with this technique.

The procedure begins by dividing the whole device into sub-components such as mechanical springs, electrostatic elements, dashpots, and proofmasses. These subcomponents are separately meshed and simulated over the desired ranges of operation. These full 3-D physics simulations are done in MEMCAD [15] using hybrid finite element and accelerated boundary element physics. The results of these simulations are fitted to multi-variable polynomials as functions of the desired degrees of freedom (DOFs). The macro-models for each subcomponent are then automatically generated in the behavioral modeling language of a system level simulator (SABER, SPICE, etc.). Finally, the component-level macro-models are assembled into a system-level design to model the behavior of the whole system.

\subsection{Implementation}

The modeling technique has been implemented in a tool named AutoMM (Auto Macro Modeler). The basic steps involve exploring the device operation space, modeling the data through multi-degree polynomial curvefitting, and using the polynomial coefficients and other simulation data in dynamic equations. AutoMM consists of several sub-modules that are used to simulate the electrostatic, mechanical and inertial behavior of MEMS components in their operation space as a function of the DOFs.

AutoMM is built around the basic functionalities of the MEMCAD software tool suite [8]. It directly uses the MEMCAD device creation and visualization methods and applies wrappers around the solver modules. AutoMM is constructed as a collection of functional sub-modules. This allows the flexible addition of components with different physical behaviors. It also allows the calculation of the behavioral data to be done in parallel, which reduces the over-all time of macro-model generation. 


\subsection{Sequence of Operations}

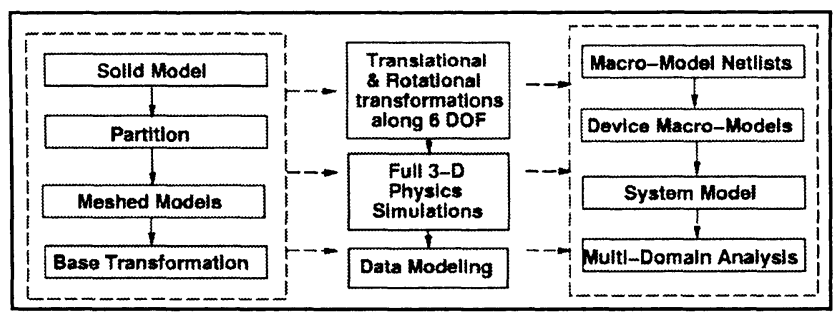

Figure 4. Sequence of operations

Figure 4 shows the sequence of operations that are carried out by the AutoMM module to generate macro-models. The procedure starts by creating the device solid model using the 'MemBuilder' module of MEMCAD [15] from the device process information and the device layout. Then a finite-element mesh is created on the solid model. This meshed solid model is input to the AutoMM module which first carries out a global base transformation on the meshed structure according to the specifications provided by the designer. Examples of such transformations include changing separations of structures, angular orientations, lengths, thicknesses, density, Poisson ratio, stress, and other geometrical and material properties of different subsets of device components. Note that this step can account for the effects of manufacturing variations in the final device macro-model.

The transformed models are then passed to the sub-modules that perform electrostatic, mechanical, and inertial simulations using multi-DOF boundary conditions. The simulation data are then fit to multi-degree polynomial equations (up to fourth order), which are functions of the degrees of freedom over which the device has been simulated. These polynomial fit coeffcients are finally used in system equations to create the device macro-model. Although most of these steps are automated, user interactions and interventions have been allowed in a few cases to include the capability of monitoring the simulation process and specification of user-defined macromodel parameters. 


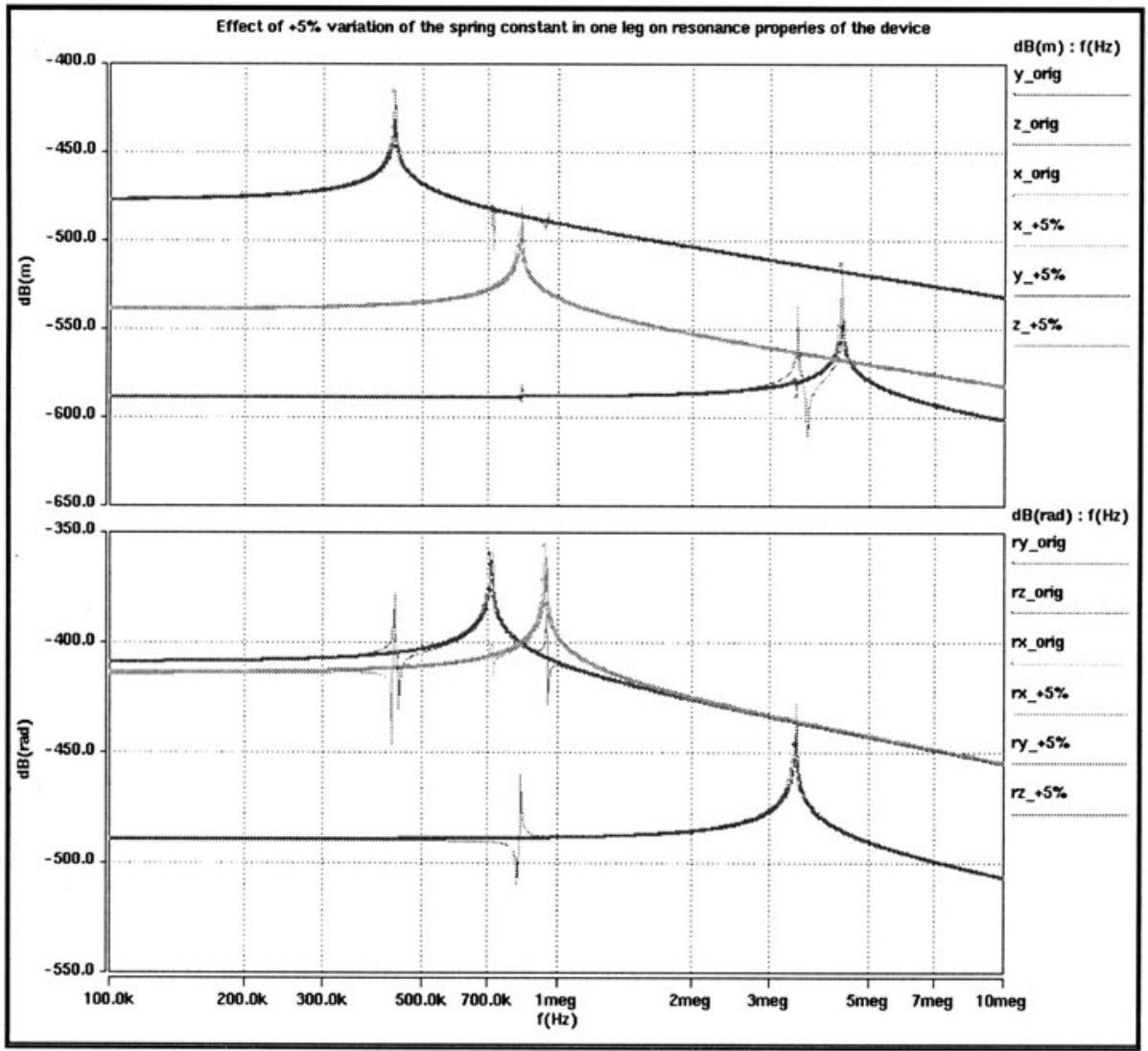

Figure 5. Frequency response in six degrees of freedom of a plate-tether MEMS structure. The thick lines show the nominal response for the 3 translational and 3 rotational DOFs. The thin lines show mode coupling with a $5 \%$ variation of thickness of one tether

Figure 5 illustrates the type of results that can be obtained using macromodels generated by AutoMM. The frequency response of a plate-tether structure is compared to that of the same structure with a variation in the thickness of a tether, which breaks the symmetry, and introduces coupling between the translational and rotational degrees of freedom. This is accomplished by modifying the spring constant of a tether, without having to extract another macro-model from a full 3-D simulation. 


\section{SUMMARY}

Development of micro-electro-mechanical systems (MEMS) products is currently hampered by the need for design aids, which can assist in integration of all domains of the design. The cross-disciplinary character of microsystems requires a top-down approach to system design which, in turn, requires designers from many areas to work together in order to understand the effects of one sub-system on another.

In this paper we have summarize some of the past three years work on developing methods and tools to enable the concurrent design of MEMS and MST systems. First we described the concurrent design methodology itself, with particular attention to who does the designing and how different designers interact. Secondly, a modeling procedure that automates the generation of macro-models of MEMS devices and shows good agreement to full 3-D physics simulation has been presented. This tool corresponds to a software realization of the thick arrow between the System Architect, and the MEMS designer in Figure 1. The implemented modeling technique is currently limited to the class of devices where the actuating and restoring forces are limited to electrostatic, mechanical (tensile and torsional), damping, and inertial types. Future developments will consider electrostatically active mechanical tethers with nonzero mass and macromodels for other physical forces, such as fluidic pressure, thermal stress, piezo-electric potential, etc.

\section{ACKNOWLEDGEMENTS}

The authors acknowledge the support of the DARPA CiMEMS Program (Contract No. F30602-96-2-0290). The support of Jonathan Hammond (Motorola SP) and Dennis Greywall (Lucent Technologies) through many discussions is greatly appreciated.

\section{REFERENCES}

1 S. D. Senturia, CAD Challenges for Microsensors, Microactuators and Microsystems, Proc. IEEE, Vol. 86, pp. 1611-1626, 1998.

2 G. Wachutka, "The Art of Modeling Coupled-Field Effects in Microdevices and Microsystems", Technical proceedings of the 1999 International Conference on Modeling and Simulation of Microsystems, MSM 99, San Juan, Puerto Rico, U.S.A., Apri19-21, 1999, pp.14-19. 
3 B. F. Romanowicz, "Methodology for the Modeling and Simulation of Microsystems", Ed. S. D. Senturia, MICROSYSTEMS, Kluwer Academic Publishers, ISBN 0-7923-8306-0, 1998.

4 R. Neul, U. Becker, G. Lorenz, P. Schwarz, J. Hasse, and S. Wünsche, "A Modeling Approach to Include Mechanical Microsystem Componets into the System Simulation", in DATE'98 - Design, Automation and Test in Europe, vol. 1, pp.510-517, Feb, 1998.

5 G. K. Fedder and R. T. Howe, "Multimode Digital Control of a Suspended Polysilicon Microstructure", Journal of Microelectromechanical Systems, pp. 283-297, May, 1996.

6 N. R. Swart, S. F. Bart, M. H. Zaman, M. Mariappan, J. R. Gilbert, and D. Murphy, "AutoMM: Automatic Generation of Dynamic Macromodels for MEMS Devices," Proc. 11th IEEE Intl. Workshop on Micro Electromechanical Systems (MEMS'98), pp. 178-183, Heidelberg, Germany, January 25-29, 1998.

7 S. F. Bart, "CAD for Integrated Surface Micromachined Sensors: Present and Future", in the Proceedings of the Fifth ACM/SIGDA Physical Design Workshop, Reston, VA, April 15-17, 1996, pp. 72-75.

8 G. Lorenz and R. Neul, "Network-Type Modeling of Micromachined Sensor Systems", Proceedings of 1998 International Conference on Modeling and Simulation of Microsystems, Semiconductors, Sensors and Actuators (MSM'98), pp. 233-238, Santa Clara, California, USA, April 6-8, 1998.

9 S. F. Bart, N. R. Swart, M. Mariappan, M. H. Zaman, and J. R. Gilbert, "An Environment for MEMS Design and Verification," Proceedings of 1998 International Conference on Modeling and Simulation of Microsystems, Semiconductors, Sensors and Actuators (MSM'98), pp. 386-391, Santa Clara, California, USA, April 6-8, 1998.

10 B. F. Romanowicz, et al., "VHDL 1076.1 Modeling Examples for Microsystem Simulation," published in "Current Issues in Electronic Modeling", Issue No. 10, Berg et al. Editors, Kluwer Academic Publishers, July 1997, ISBN 0-7923-9875-0.

11 S. Sherman, et al., "A Low Cost Monolithic Accelerometer," Proc. IEEE 1992 Int. Elec. Dev. Meeting, San Francisco, CA, Dec. 13-16, 1992, pp. 19.1.1-19.1.4.

12 L. Spangler and C. J. Kemp, "ISAAC - Integrated Silicon Automotive Accelerometer," Proc. of the 8th International Conf. Of Solid-State Sensors and Actuators, June 25-29, 1995, pp. 585-588.

13 S. F. Bart and H. R. Samuels, "Design Techniques for Minimizing Manufacturing Variations in Surface Micromachined Accelerometers," Microelectromechanical Systems, ASME, DSC-Vol. 59, 1996, pp. 427-433.

14 M. H. Zaman, S. F. Bart, V.L. Rabinovich, C.K. Ghaddar, I. Tchertkov, and J. R. Gilbert, "A Technique for Extraction of Macro-Models in System Level Simulation of Inertial Electro-Mechanical Micro-Systems," Technical Proceedings of 1999 International Conference on Modeling and Simulation of Microsystems, MSM 99, pp. 163-167,San Juan, Puerto Rico, USA, April 19-21, 1999.

15 M. H. Zaman, S. F. Bart, V.L. Rabinovich, C.K. Ghaddar, I. Tchertkov, and J. R. Gilbert, "A Technique for Extraction of Macro-Models in System Level Simulation of Inertial Electro-Mechanical Micro-Systems," Technical Proceedings of 1999 International Conference on Modeling and Simulation of Microsystems, MSM 99, pp. 163-167,San Juan, Puerto Rico, USA, April 19-21, 1999. 